\title{
Cervicogenic Vertigo Treated by C1 Transverse Foramen Decompression: A Case Report
}

\author{
Junhee Park, Chulkyu Lee, Namkyu You, Sanghyun Kim, Kihong Cho \\ Department of Neurosurgery, Ajou University School of Medicine, Suwon, Korea
}

Cervicogenic vertigo was known as Bow hunter's syndrome. Occlusion of vertebral artery causes vertebrobasilar insufficiency and we reported cervicogenic vertigo case which was treated by simple decompression of transverse foramen of C1. The patient was 48 years old female who had left side dominant vertebral artery and vertigo was provoked when she rotated her head to right side. Angiography showed complete obliteration of blood flow of left vertebral artery when her head was rotated to right side. The operation was decompression of left vertebral artery at $\mathrm{C} 1$ level. Posterior wall of transverse foramen was resected and vertebral artery was exposed and decompressed. After surgery, vertigo of the patient was disappeared, and angiography showed patent left vertebral artery when her head was rotated to right side. Vertigo caused by compression of cervical vertebral artery could be treated by decompression without fusion or instrumentation, especially in $\mathrm{C} 1$ transverse foramen.

Key Words: Vertigo $\cdot$ Vertebrobasilar insufficiency $\cdot$ Vertebral artery $\cdot$ Transverse foramen $\cdot$ Decompression

\section{INTRODUCTION}

Vertigo is a common symptom that physicians may meet in their clinical activities. Vertigo is sometimes associated with intracranial lesion or ontological lesion. Rarely, vertigo may be caused by cervical spine lesion which is known as Bow hunter's syndrome ${ }^{1)}$. Bow hunter's syndrome is symptomatic vertebrobasilar insufficiency (VBI) caused by occlusion of the vertebral artery in cervical spine which is provoked by head rotation $^{2,6,11,12)}$. Typical symptoms include diplopia, dysarthria, visual disturbances, nystagmus, vertigo and ataxia ${ }^{5,7}$. Several reports suggested various treatment methods such as conservative management, surgical decompression of vertebral artery and endovascular treatment ${ }^{2}$. We report a patient with classical bow hunter's syndrome who was treated by decompression of $\mathrm{C} 1$ transverse foramen.

- Received: June 27, 2014 - Revised: August 25, 2014

- Accepted: September 1, 2014

Corresponding Author: Namkyu You, MD

Department of Neurosurgery, Ajou University School of Medicine,

164 Worldcup-ro, Youngtong-gu, Youngtong-gu, Suwon 442-731, Korea

Tel: +82-31-219-5230, 5237, Fax: +82-31-219-5238

E-mail:nkyou@ajou.ac.kr

®This is an Open Access article distributed under the terms of the Creative

Commons Attribution Non-Commercial License (http://creativecommons.org/ licenses/by-nc/3.0/) which permits unrestricted non-commercial use, distribution, and reproduction in any medium, provided the original work is properly cited.

\section{CASE REPORT}

A 48 years old female visited with repeated vertigo attack which had emerged when she rotated her head to right side. She also complained of tinnitus on left side simultaneously. She had otitis media at left ear in the past but treated well and vestibular test showed normal result. On physical examination there was no neurologic deficit in neutral position, but left side nystagmus was found when her head was rotated. Computed tomographic angiography (CTA) showed that left vertebral artery was dominant and right vertebral artery was hypoplastic (Fig. 1). Angiography was performed in supine position. Arterial filling and patency was intact in neutral position. But in right side head rotation position, the patient experienced vertigo attack and angiography showed occlusion of left vertebral artery (Fig. 2). Surgical decompression was performed. After induction of general anesthesia, the patient

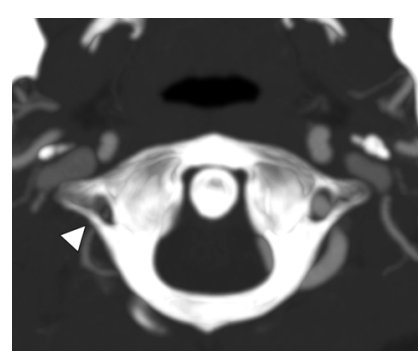

Fig. 1. Preoperative computed tomographic angiography at $\mathrm{Cl}$ vertebra level in axial view. Right vertebral artery was hypoplastic than left side one (Arrowhead). 
was positioned in prone position and her head was mildly flexed. Midline vertical was incised and C1 posterior arch \& C2 spinous process was exposed. A part of left posterior arch and transverse foramen of $\mathrm{C} 1$ were removed by kerrison rounguer and high speed drill (Fig. 3). Posterior side of vertebral artery was exposed and its pulsatile movement was observed after decompression. Gelfoam was applied on the decompressed site and wound was closed layer by layer. Immediately after surgery, the patient's symptoms were decreased. Vertebral artery angiography was performed 1 month later and occlusion of left vertebral artery during right side head rotation was not

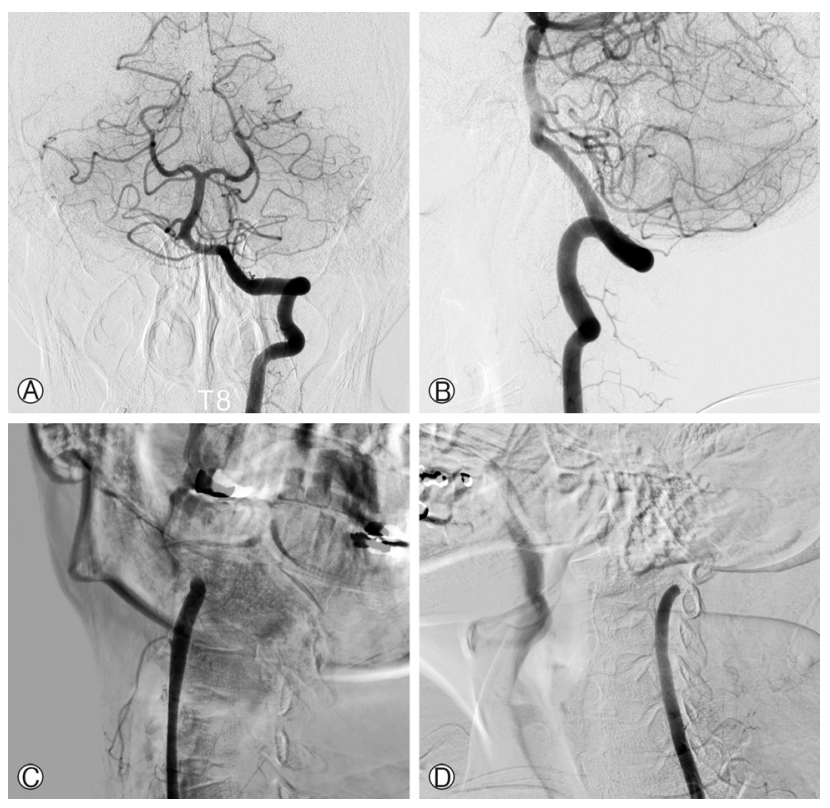

Fig. 2. Cerebral angiography in neutral position and rotation of head to right position. Patency of left vertebral artery flow was seen in AP view (A) and lateral view (B). When the patient's head was rotated to the right side, flow of vertebral artery was obstructed at $\mathrm{Cl}$ transverse foramen level in $\mathrm{AP}$ view (C) and lateral view (D). observed anymore (Fig. 4). The patient did not have vertigo attack again.

\section{DISCUSSION}

Dizziness and vertigo is frequently met in clinical activities of neurosurgeons and there are many causes. In rare cases, these symptoms may be caused by vertebral artery occlusion at cervical spine and causes VBI. Numerous causes of VBI are reported of which the most common cause is arthrosclerosis of the arteries distributed in the brain stem ${ }^{1)}$. Osteophytes, cervical musculature, fascial bands, anomalies of cervical spine and vertebral artery are other extracranial mechanical causes of $\mathrm{VBI}^{1,9)}$. Most of reported cases in bow hunter's syndrome showed occlusion of a dominant vertebral artery due to mechanical stretching or compression and dislodgment between C12 with head rotation ${ }^{8}$. Our case did not show above features

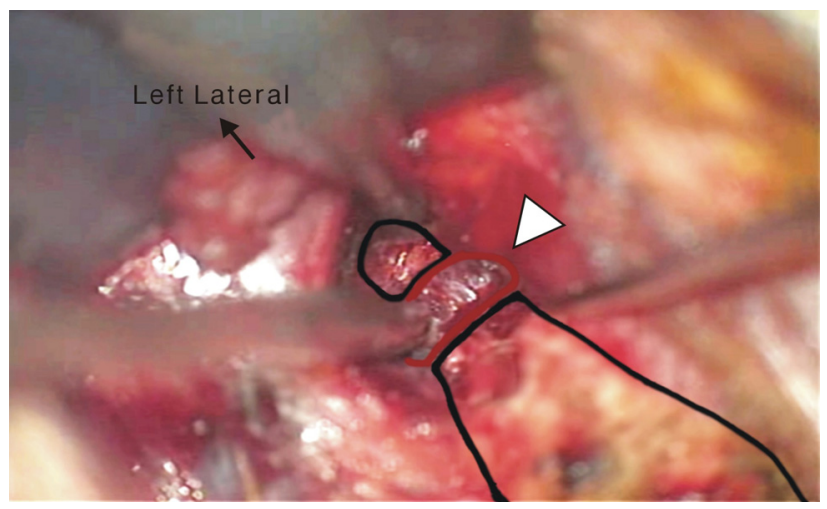

Fig. 3. Intraoperative microscopic view. Removal of posterior bony structure of $\mathrm{Cl}$ left transverse foramen. Black line indicate left side of posterior arch of $\mathrm{Cl}$ and red line indicated left vertebral artery. Vertebral artery was decompressed well (Arrow head).
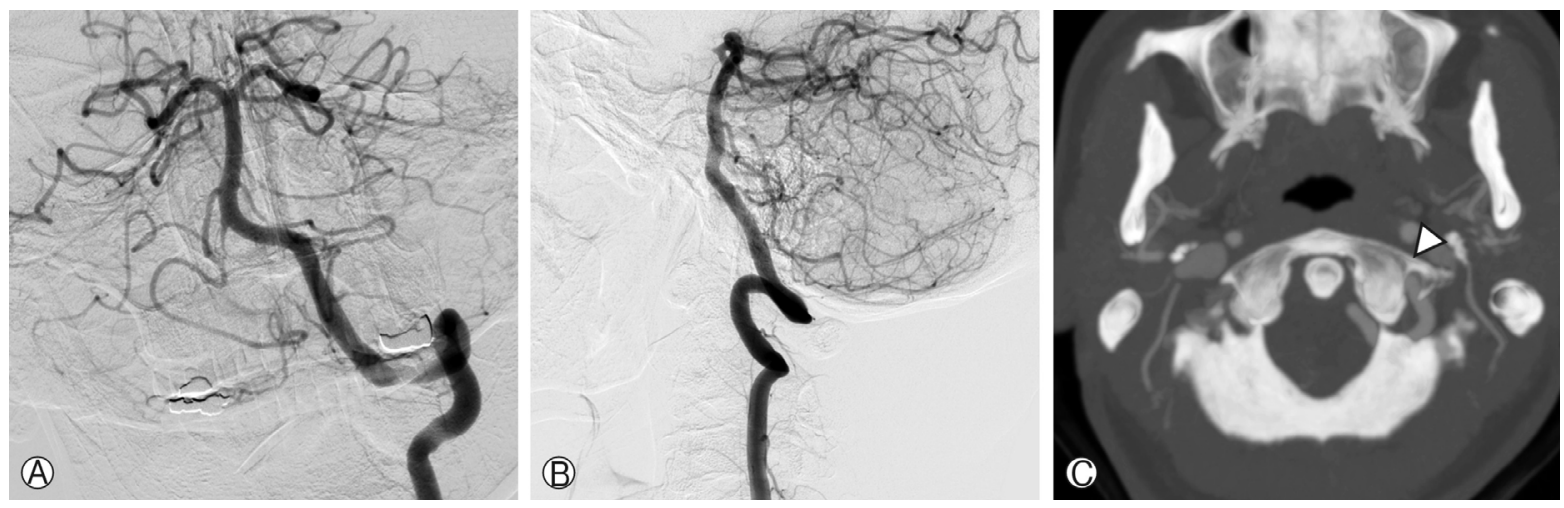

Fig. 4. Postoperative angiography and CT angiography. Blood flow was maintained in head rotation position (A and B). Posterior wall of left transverse foramen was resected (Arrow head) (C). 
but occlusion of vertebral artery was limi- ted in transverse foramen of atlas. This bow hunter's syndrome was difficult to diagnose without suspicion of cervical spine lesion of vertebral artery ${ }^{2,4,9}$.

Gold standard for diagnosis of bow hunter's syndrome is conventional angiography in various motion of cervical spine ${ }^{2)}$. This case showed complete occlusion of blood flow of left vertebral artery when the head was rotated to right side. Computed tomography angiography is needed to understand structural correlation between vertebral artery and bony component of cervical spine. Magnetic resonance image also may be helpful for identifying ischemic change of cerebellar parenchyma or stability of cervical spine. Transcranial Doppler examination may be needed to check blood flow of vertebral artery without radiation exposure.

Mechanical decompression of vertebral artery is the most favorable treatment goal for bow hunter's syndrome. In mild cases, conservative treatment such as cervical brace appliance, limitation of neck motion and anticoagulation medication is helpful for symptom relief. Surgical decompression may be considered when these conservative methods fail. Numerous literatures report decompression of vertebral artery with or without fusion surgery achieved favorable outcome ${ }^{6,8,10,13)}$. But there was limitation of neck motion after fusion surgery, especially in C1-2 fusion. Takeshi reported total relief of vertigo in fusion surgery patients but only $66.7 \%$ relief of symptoms in decompression surgery patients ${ }^{8}$. This may be due to complex structure of C1-2 lesion. Surgical methods should be determined after careful evaluation. Other treatment option is endovascular surgery. Darkhabani et al reported 4 cases of bow hunter's syndrome and vertebral artery stenting at V2 segment (C2-C6 level) had been achieved complete relief of their symptoms ${ }^{3}$. Our case was treated with decompression only of left side transverse foramen of $\mathrm{C} 1$ where mechanical compression had occurred, and the patient was relieved from symptoms without limitation of cervical spine mobility. If compromise of vertebral artery is limited in bony structure of transverse foramen, direct decompression without fusion or screw fixation may be the best option for preserving cervical spine motion.

\section{CONCLUSION}

Vertigo caused by head rotation may be suspected as vertebral artery insufficiency such as bow hunter's syndrome. Angiography is essential for diagnosis and decision of surgical treat- ment and adequate decompression of dominant vertebral artery without fusion surgery could achieve favorable outcome.

\section{REFERENCES}

1. Barton J, Margolis M: Rotational obstruction of the vertebral artery at the atlantoaxial joint. Neuroradiology 9(3):117-120, 1975

2. Cornelius JF, George B, Oka DNd, Spiriev T, Steiger HJ, Hänggi D: Bow-hunter's syndrome caused by dynamic vertebral artery stenosis at the cranio-cervical junction-a management algorithm based on a systematic review and a clinical series. Neurosurgical Review 35(1):127-135, 2012

3. Darkhabani MZ, Thompson MC, Lazzaro MA, Taqi MA, Zaidat OO: Vertebral artery stenting for the treatment of bow hunter's syndrome: Report of 4 cases. J Stroke Cerebrovasc Dis 21(8):908 e901-905, 2012

4. Iguchi Y, Kimura K, Shibazaki K, Iwanaga T, Ueno Y, Inoue T: Transcranial doppler and carotid duplex ultrasonography findings in bow hunter's syndrome. Journal of Neuroimaging 16(3): 278-280, 2006

5. Kim H-A, Yi H-A, Lee C-Y, Lee H: Origin of isolated vertigo in rotational vertebral artery syndrome. Neurological Sciences 32(6):1203-1207, 2011

6. Kim K, Isu T, Morimoto D, Kominami S, Kobayashi S, Teramoto A: Anterior vertebral artery decompression with an ultrasonic bone curette to treat bow hunter's syndrome. Acta Neurochirurgica 150(3):301-303, 2008

7. Kuether TA, Nesbit GM, Clark WM, Barnwell SL: Rotational vertebral artery occlusion: A mechanism of vertebrobasilar insufficiency. Neurosurgery 41(2):427-433, 1997

8. Matsuyama T, Morimoto T, Sakaki T: Comparison of c1-2 posterior fusion and decompression of the vertebral artery in the treatment of bow hunter's stroke. Journal of Neurosurgery 86 (4): 619-623, 1997

9. Piñol I, Ramirez M, Saló G, Ros AM, Blanch AL: Symptomatic vertebral artery stenosis secondary to cervical spondylolisthesis. Spine 38(23):E1503-E1505, 2013

10. Seki T, Hida K, Akino M, Iwasaki Y: Anterior decompression of the atlantoaxial vertebral artery to treat bow hunter's stroke: Technical case report. Neurosurgery 49(6):1474-1476, 2001

11. Sorensen BF: Bow hunter's stroke. Neurosurgery 2(3):259-261, 1978

12. Whitmore RG, Simon SL, Hurst RW, Nisenbaum HL, Kasner SE, Zager EL: Bow hunter's syndrome caused by accessory cervical ossification: Posterolateral decompression and the use of intraoperative doppler ultrasonography. Surgical Neurology 67 (2): 169-171, 2007

13. Yang PJ, Latack JT, Gabrielsen T, Knake J, Gebarski S, Chandler W: Rotational vertebral artery occlusion at c1-c2. AJNR Am J Neuroradiol 6(1):96-100, 1985 